\title{
A multidisciplinary group for prostate cancer management: A single institution experience
}

\author{
ANTONELLA COLASANTE $^{1}$, ANTONIETTA AUGURIO ${ }^{2}$, RAFFAELLA BASILICO ${ }^{3}$, \\ ANTONIO RAFFAELE COTRONEO ${ }^{3}$, MARIA B. DI SCIASCIO ${ }^{4}$, GIUSEPPE GASPARI ${ }^{5}$, DOMENICO GENOVESI $^{2}$, \\ JAMARA GIAMPIETRO $^{6}$, ROMINA IANTORNO ${ }^{5}$, ANTONIO MARCHETTI ${ }^{1}$, CLARA NATOLI $^{6}$, \\ ANTONELLO NUZZO $^{6}$, FLORIANA OTTAVIANI $^{7}$, PAOLO POMPA $^{8}$, PIETRO CASTELLAN $^{5}$, \\ RAFFAELE TENAGLIA ${ }^{9}$ and ANNAMARIA VINCIGUERRA ${ }^{2}$
}

\author{
Departments of ${ }^{1}$ Surgical Pathology, ${ }^{2}$ Radiotherapy, ${ }^{3}$ Radiology, ${ }^{4}$ Clinical Governance, ${ }^{5}$ Urology, ${ }^{6}$ Oncology and \\ ${ }^{7}$ Nursing Management, SS. Annunziata Hospital, I-66100 Chieti; ${ }^{8}$ Chief Urological Unit, Casa di Cura Privata Pierangeli, \\ I-65124 Pescara; ${ }^{9}$ School of Medicine, G. D'Annunzio University, I-66100 Chieti, Italy
}

Received October 18, 2016; Accepted October 17, 2017

DOI: $10.3892 / \mathrm{ol} .2017 .7506$

\begin{abstract}
Increased knowledge regarding the heterogeneity of Prostate Cancer (PC) and its variable outcomes has generated controversy over the best clinical approach. Nowadays, it is well-known that patient outcomes and clinical management may be improved by an efficient organization of the national health care system. The Interdisciplinary Group for Oncological Care (GICO) for PC patients was created by our healthcare management company in September 2010. Since then, a multidisciplinary internal report was applied to PC patients. This report highlights our methodology and experience of planning a GICO, and illustrates the results obtained for the management of PC patients before and after the adopted GICO criteria in a single institution, the SS Annunziata Hospital (Chieti, Italy).
\end{abstract}

\section{Introduction}

Prostate cancer (PC) is the second leading cause of cancer death in males and is the most frequently diagnosed cancer in men. Despite this, the prostate cancer mortality remains stable over time. Deaths for cancer could be prevented not only through primary and secondary prevention, but also through timely interventions in terms of diagnosis and treatment, in the context of appropriate diagnostic and therapeutic care (1-4).

Moreover in Italy PC is the prevalent cancer in men. In 2011, 7,520 men died due to PC (about $8 \%$ of total deaths from cancer)

Correspondence to: $\operatorname{Dr}$ Antonella Colasante, Department of Surgical Pathology, SS. Annunziata Hospital, Via dei Vestini, I-66100 Chieti, Italy

E-mail: acolasante@unich.it

Key words: prostate cancer, multidisciplinary team, shared decision making and this neoplasm is the third cause of cancer death after lung (27\%) and colon-rectum cancers (10\%) (2).

Increased knowledge regarding the heterogeneity of PC and its variable clinical course resulted in controversy over the best therapeutic approach. Physicians have to exhaustively inform their patients about their own disease and their own targeted therapeutic options: They have to balance between diagnostic and therapeutic uncertainty and, on the other side, overtreatment. Moreover, they have to take into account the long clinical course of PC and possible co-morbidity. Many patients are more confident in following a shared medical decision (3). Other important aspects need to be added: The patients' reaction facing a multidisciplinary team (MDT), how this MDT is able to manage the disease, and which grade of satisfaction the patients can get. Furthermore, efficient organization of the national healthcare system can be an additional instrument for improving patient's outcome.

For all these reasons there is a need to establish a MDT approach. In this model, patient care is step by step coordinated by specialists from multiple disciplines which are involved in a decision-making process based on evidence based medicine principles. Other advantages for the patients include best clinical outcome, better patient satisfaction and compliance. All MDT participants have excellent educational and professional experience; they also have the possibility to recruit patients for clinical trials (5).

This report highlights our methodology and experience to plan a decision-making model based on an Interdisciplinary Group of Oncological Care named GICO and illustrates results obtained for the management of PC from January 2007 to December 2010 (cohort 1, without GICO) and from January 2011 to December 2014 (cohort 2, with GICO) in Chieti SS Annunziata Hospital.

\section{Patients and methods}

A GICO for PC was implemented under the coordination of the Clinical Governance Staff of the Chieti Hospital 
Managership in September 2010 and included urologists, radiologists, radiation therapists, medical oncologists, and histopathologists. From September 2010 to January 2011, the Group used the following methodology for the drafting of an internal document with diagnostic and therapeutic guideline.

Reference guidelines were drafted using the SIGN (Scottish Intercollegiate Guidelines Network) and the GRADE method (http://www.sign.ac.uk). Together with the guidelines of the Italian Association of Urologists, European Association of Urology (EAU Guidelines), National Institute for Health and Care Excellence (NICE, UK), and American Association of Urology (USA) and considering local health care system availability.

A multidisciplinary internal report (GICO document) was drafted with a flowchart of clinical evidence, and an organizational route timing was elaborated.

A matrix of responsibilities for each area of specialty has been developed and process indicators (histological diagnosis in $<10$ days, prostatectomy in less than 45 days, radiotherapy in less than 180 days), based on Italian healthcare guidelines for internal institutional audit evaluations, have been defined, with the expectation to provide, further on, the outcome indicators. At that time, the three-year National Health Plan of the Ministry of Health established that cancer must be treated by surgery within 30 days from the diagnosis and incorporated under the law in Regione Abruzzo (http://bura.regione.abruzzo.it/2011/Allegati/Spe_55_01_All_ A_DGR_575, table on page 68).

The patients (and family members with the consensus of the patient) were informed face to face by all the GICO professionals. Consultation contained a full overview of the possible therapeutic options available in our hospital as well as the information about the individual risk profile determined at the time of consultation: Patient's age and co-morbidities, medications, clinical palpation findings, PSA levels, Gleason Score of biopsy, number of positive biopsy cores, CT- or MRI-scan.

In order to evaluate the impact of the meetings on clinical management, data from all the patients who received radical treatment with surgery or radiotherapy (with or without androgen deprivation therapy, ADT) for PC in Chieti's Hospital from January 2007 to December 2010 (cohort 1) and from January 2011 to December 2014 (cohort 2) were analyzed. Cohort 1 had no interdisciplinary consultation, Cohort 2 had GICO consultation for PC.

In order to evaluate patient satisfaction we developed a questionnaire covering: Quality of information given by the team, care by the physician staff, care by the Case Manager. The overall level of satisfaction was determined by asking the patients if they would recommend the consultation of the interdisciplinary group for prostate cancer of Chieti's Hospital to people with prostate cancer. Patients were asked to fill in the questionnaire anonymously at home and return it to the next meeting. Patients specified their level of agreement or disagreement on a typical five-level agree-disagree Likert scale (1, strongly disagree; 2 , disagree; 3 , neither agree nor disagree; 4 , agree; 5 , strongly agree). Thus, the range captures the intensity of their feelings for a given item. The correlations between the different variables of the questionnaire were evaluated with the correlation test for Spearman's ranks ( $\rho$ ).

\section{Results}

The MDT identified the EAU guidelines using Appraisal of Guidelines for Research and Evaluation (AGREE) tool, which relied on a sequential assessment of the guidelines quality $(6,7)$.

The multidisciplinary team drafted a document reporting the clinical evidence for PC. Patient management started with histological diagnosis, included the necessary imaging studies and indicated therapeutic options for low, intermediate, high and metastatic risk group of PC. For the diagnosis, an ultrasound trans-peritoneal biopsy was performed and histological diagnosis was carried out with a centralized review method of the biopsy specimens. The flow-chart of clinical evidence is shown in Fig. 1. The complete GICO document is available on the official hospital website: www.asl2abruzzo.it and includes all therapeutic algorithms and the addendum on protocols and procedures specific for each medical discipline.

The Case Manager, a skilled nurse who took care of appointments, collection of medical tests and planned for each type of therapeutic intervention, played a very important role in the multidisciplinary team. The Case Manager followed the patient at every step of the diagnostic and therapeutic process and coordinated all activities of the MDT. Patients were enrolled in the GICO process through the Case Manager by the Urology Office, by General Physicians and by hospital departments (Fig. 2). Since 2011, the MDT met weekly at the Hospital starting with a Tumor Board approximately two hours long, where cases were discussed. The patient was then informed about different therapeutic approaches and participated on the final therapeutic option decision. The consultation time was variable and depended, among others, on the individual questions of the patient or of his family members and varied averagely between 15 to $40 \mathrm{~min}$.

The GICO activities were monitored every 6 months by internal institutional audit. From 2011 to 2014, interdisciplinary consultations supported by the GICO have been provided to 1144 PC patients coming from the SS Annunziata Hospital and the surrounding peripheral hospitals, as reported in the last institutional audit.

Seventy-four percent of PC patients in the institutional files were included in the GICO pathway and all of them followed the established program. Particularly, in the SS Annunziata Hospital of Chieti, we evaluated and proposed the appropriate therapeutic option to 412 patients. The prescribed therapeutic options were: Surgery (S) in 143 patients, Radiotherapy (RT) Alone or combined with androgen deprivation therapy (ADT) in 139 patients, ADT alone in 49 patients, Active Surveillance (AS) in 73 patients (Fig. 3). Only twenty-five percent of patients selected for surgery had surgical prostatectomy in less than 45 days. It was justified by the local internal organization and also by the fact that in low risk patients the interval may be longer.

Patients who received radical treatment by means of surgery or radiotherapy (with or without androgen deprivation therapy) from January 2007 to December 2010 (cohort 1) were 268 and from January 2011 to December 2014 (cohort 2) were 282. In cohort 1,176 patients $(65.7 \%)$ were treated by surgery and 92 patients $(34.3 \%)$ were treated by radiotherapy with or without ADT. In cohort 2, 143 patients (50.7\%) were treated by 


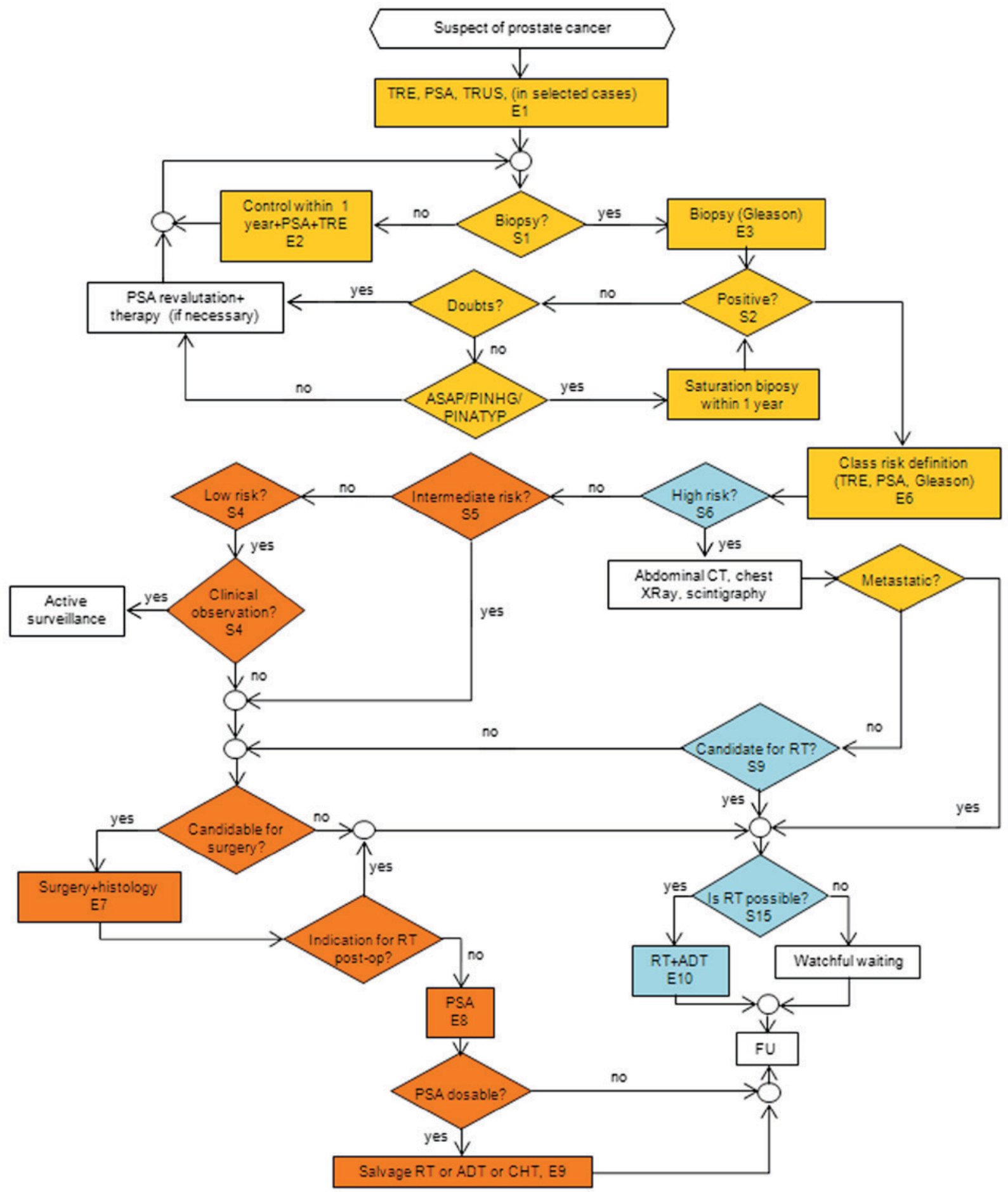

Figure 1. Clinical reasoning flowchart of the Interdisciplinary Group of Oncological Care. TRE, Trans-rectal exploration; PSA, prostatic specific antigen; TRUS, transrectal ultrasound; ASAP, atypical small acinar proliferation; PINHG, prostatic intraepithelial neoplasia high grade; PINATYP, atypical PIN; AS, active surveillance; CT, computer tomography; RT, radiotherapy; ADT, androgen deprivation therapy; CHT, chemotherapy; FU, follow-up.

surgery and 139 patients (49.3\%) were treated by radiotherapy with or without ADT.

The satisfaction questionnaire was distributed to 276 consecutive patients evaluated by the GICO from February 2013 to December 2014: 193 out of 276 patients completed and delivered it $(69,9 \%)$ The quality of information given by the team was considered satisfactory in $93.9 \%$ of cases; $84.3 \%$ of patients appreciated the care by the physician staff and $93.5 \%$ appreciated the care by the Case Manager. Ninety-seven points two percent of patients declared that the team treated them with humanity and respect and $89.7 \%$ trusted the healthcare personnel. $95.4 \%$ of patients would have recommended the consultation of the interdisciplinary group for prostate cancer of Chieti's Hospital to people with prostate cancer, and the correlation test for Spearman's ranks highlighted a high level of association with the appreciation of the care of a multidisciplinary group $(\rho, 0.850 ; \mathrm{P}<0.0001)$ and of the information received $(\rho, 0.771 ; \mathrm{P}<0.0001)$. 


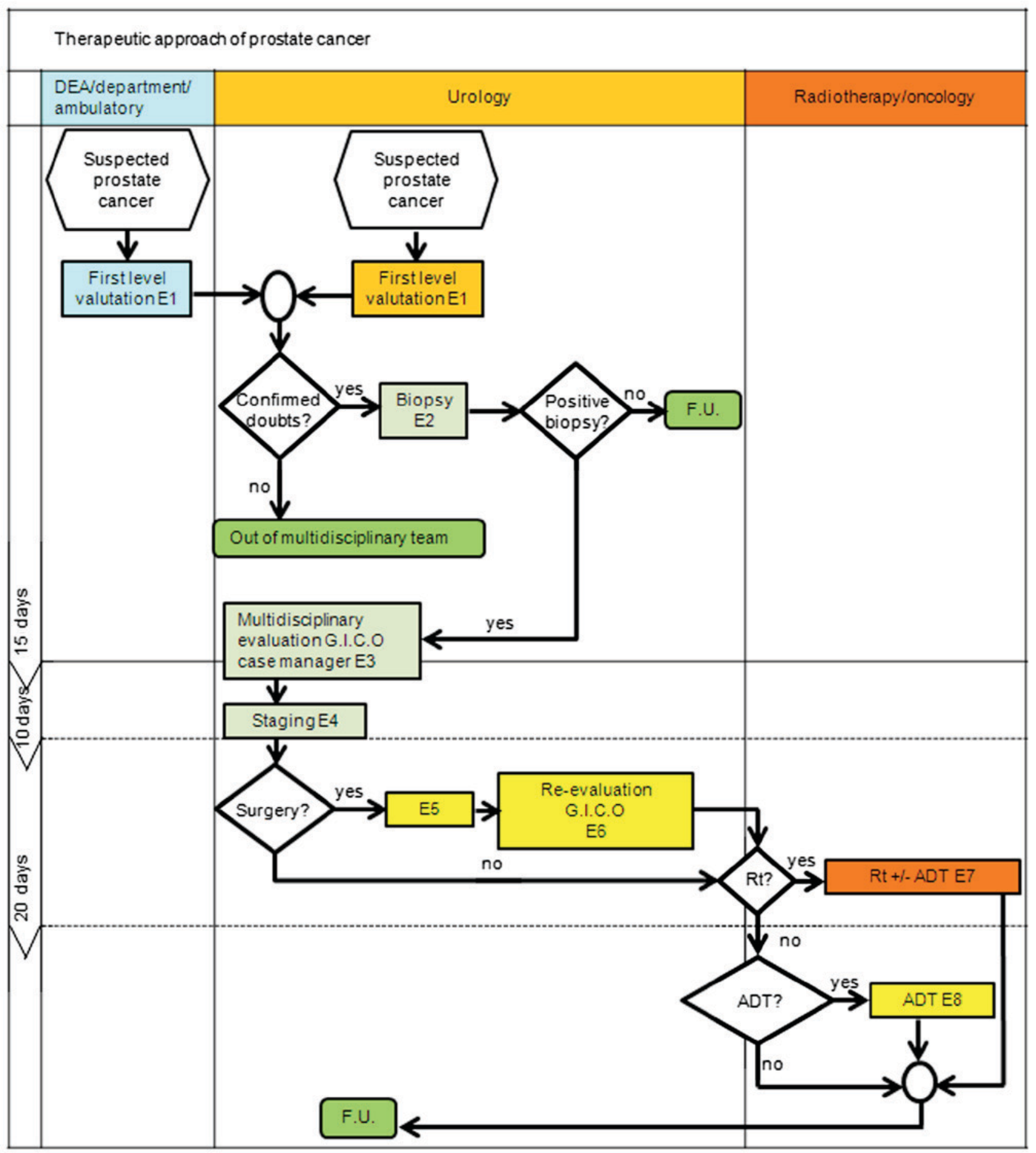

Figure 2. Organizational route timing. Rt, Radiotherapy; ADT, Androgen deprivation therapy; FU, Follow-up; GICO, Interdisciplinary Group of Oncological Care.

\section{Discussion}

$\mathrm{PC}$ is considered the most common cancer in elderly males ( $>70$ years of age) in Europe. It is a major health concern, especially in developed countries with their greater proportion of elderly men in the general population. The incidence is highest in Northern and Western Europe ( $>200$ per 100,000), while rates in Eastern and Southern Europe have showed a continuous increase. There is still a survival difference between men diagnosed in Eastern Europe and those in the rest of Europe. Overall, during the last decade, the 5-year relative survival percentages for PC steadily increased from $73.4 \%$ in $1999-2001$ to $83.4 \%$ in $2005-2007$. Early detection of prostate cancer reduces specific cancer and overall mortality and improves men's future quality of life because it decreases the risk of being diagnosed locally advanced stage or metastatic disease. Moreover, in the last years, there was a greater variety of treatment recommendations due to new therapeutic options like AS and HIFU (4; http://www.uroweb. org/guidelines/Guidelines on Prostate Cancer). 


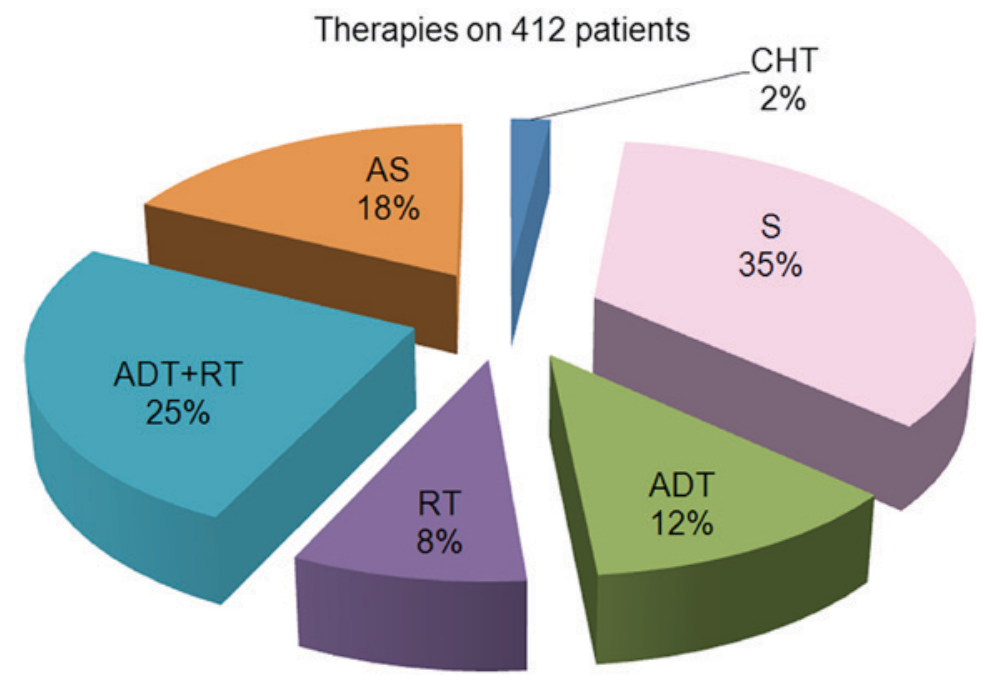

Figure 3. Patients therapies accordingly to EAU guidelines risk grouping and patient preferences. AS (light brown), Active surveillance; CHT (blue), Chemotherapy; ADT (green), Androgen deprivation therapy; RT (violet), Radiotherapy; S (pink), Surgery; ADT + RT (light blue) (percentage of total patient number).

We established a GICO pathway and evaluated 1144 PC patients; specifically in SS Annunziata Hospital we treated 412 patients according to our multidisciplinary model.

In the Italian healthcare system, the institution of the MDTs is considered a gold standard in cancer therapy and care. In this regard, there are published studies on the MDTs that show that it may ensure more appropriate treatment through a full preoperative review of imaging and pathology reports and that a multidisciplinary approach guarantees the most up-to-date treatment for all PC patients. Other clinical-based goals included improving patients' quality of life and setting up a structured follow-upcareplan. As ithappensinourHospital, there is a weekly scheduled day in which the MDT meet, with simultaneous data evaluation, management discussion, and patient consultations from a multidisciplinary team of PC specialists, and this is feasible $(8,9)$.

As underlined above, in our institution all the members of the GICO team meet weekly in order to discuss clinical cases and to inform directly the patients about the diagnostic and therapeutic approach. From our data in Fig. 3, the primary therapies in cohort 2 were distributed between surgery (35\%) and radiotherapy (33\%). We offered as primary treatment active surveillance in all low risk PC cases (18\%); however the percentage of cases who accepted was very low, mostly because of reported psychological stress (less than $2 \%$, data not shown). In cohort 1 there was a prevalence in surgical treatment $65,7 \%$ vs. radiotherapy $34,3 \%$. Primary therapies were more distributed by comparing cohort 1 and 2.

This kind of selective approach for cancer care provided the patients with the opportunity to learn about all diagnostic and therapeutic options and to discuss the recommendations of their physicians in an open and interactive mode. This approach encouraged a shared decision-making and potential reduction in physician bias. The same benefits have been reported in other papers with reference to different oncological diseases. The papers showed that patients with other cancers, different from PC, managed by MDT, may have a better outcome $(4,9-14)$.
One of the advantages described by our patients about the GICO team was the fact that they were actively involved in the therapeutic decision with all the GICO professionals; good results have been achieved on improved accessibility and perception in communication with the medical team. Last, but not least, this approach has been successful in reducing delays to conclude the diagnostic and therapeutic process. This was assessed also by the internal institutional audit evaluating the process indicators.

Taking into account the recent literature, we are modifying the clinical flow chart schedule, for instance, performing multi-parametric magnetic resonance imaging (MP-MRI) and TRUS fusion biopsy in patients with progressive increase in PSA levels and negative TRUS biopsies. In fact, MP-MRI allows to detect more cases of clinically significant cancer compared with the standard TRUS-biopsy (15), avoiding a primary biopsy and diagnosis of some clinically insignificant cancers.

The establishment of Prostate Cancer Units could favor the best clinical practice avoiding inappropriate procedures, deliver high-quality care to patients and, may be, providing financial saving. These aspects are particularly relevant considering the high-incidence of PC. However, there is lack of evidence indicating that MDT approach resulted in improvements in clinical outcomes because studies are retrospective, with poor sample size $(12-14,16)$.

Although our data are pro establishment of MDT on PC, future research should assess the impact of MDT approach on patient satisfaction and quality of life, as well as, rates of cross-referral between disciplines $(4,15)$.

We created a sort of local chain, a mutual support professional's network for the patients, interested in all aspects of Evidence Based Healt Care (EBHC), that provided Contact, Help, Advice, Information in a Network.

In conclusion, even Munro et al recently stated that MDT approach have outlived their usefulness (16), we can say that the MDT approach needs to move forward. Moreover, our results suggest that the future of PC patients relies in a successful multidisciplinary collaboration between 
experienced physicians. This may lead to important progress in all the phases and aspects of PC management.

\section{References}

1. Siegel RL, Miller KD and Jemal A: Cancer Statistics, 2015. Ca Cancer J Clin 65: 5-29, 2015.

2. Ferlay J, Soerjomataram I,Dikshit R, Eser S, Mathers C, Rebelo M, Parkin DM, Forman D and Bray F: Cancer incidence and mortality worldwide: Sources, methods and major patterns in GLOBOCAN 2012. Int J Cancer 136: E359-: E386, 2015.

3. Baumunk D, Reunkoff R, Kushner J, Baumunk A, Kempkensteffen C, Steiner U, Weikert S, Moser L, Schrader M, Höcht $\mathrm{S}$, et al: Interdisciplinary decision making in prostate cancer therapy-5-years' time trends at the interdisciplinary prostate cancer center (IPC) of the Charité Berlin. BMC Med Inform Decis Mak 13: 83, 2013.

4. Sciarra A, Gentile V and Panebianco V: Multidisciplinary management of prostate cancer: How and why. Am J Clin Exp Urol 1: 12-17, 2013.

5. Genovesi D, Mazzilli L, Trignani M, Di Tommaso M, Nuzzo A Biondi E, Tinari N, Martino MT, Innocenti P,DI Sebastiano P, et al: Developing a decision-making model based on an interdisciplinary oncological care group for the management of colorectal cancer. Anticancer Res 34: 2525-2531, 2014.

6. AGREE Collaboration: Development and validation of an international appraisal instrument for assessing the quality of clinical practice guidelines: The AGREE project. Qual Saf Health Care 12: 18-23, 2003.

7. Brouwers MC, Kho ME, Browman GP, Burgers JS, Cluzeau F, Feder G, Fervers B, Graham ID, Grimshaw J, Hanna SE, et al: AGREE next steps consortium: AGREE II: Advanced guideline development, reporting and evaluation in health care. CMAJ 182: E839-E842, 2010.

8. Prades J, Remue E, van Hoof E and Borras JM: Is it worth reorganizing cancer services on the basis of multidisciplinary teams (MDTs)? A systematic review of the objectives and organization of MDTs and their impact on patient outcomes. Healthpol Policy 119: 464-474, 2015
9. Sundi D, Cohen JE, Cole AP, Neuman BP, Cooper J, Faisal FA, Ross AE and Schaeffer EM: Establishment of a new prostate cancer multidisciplinary clinic: Format and initial experience. Prostate 75: 191-199, 2015.

10. Bayoud Y, Loock PY, Messaoudi R, Ripert T, Pierrevelcin J, Kozal S, Leon $\mathrm{P}$, Kamdoum $\mathrm{M}$, Irène $\mathrm{C}$ and Menard $\mathrm{J}$ : Prostate cancer: What about reproducibility of decision made at multidisciplinary team management? Urol J 12: 2078-2082, 2015.

11. Khani $\mathrm{MH}$ and Smedh $\mathrm{K}$ : Centralization of rectal cancer surgery improves long-term survival. Colorectal Dis 12: 874-879, 2010.

12. Morris E, Haward RA, Gilthorpe MS, Craigs C and Forman D: The impact of the Calman-Hine report on the processes and outcomes of care for Yorkshire's colorectal cancer patients. Br J Cancer 95: 979-985, 2006.

13. Wilks JA, Liebig C, Tasleem SH, Haderxhanaj K, Lee L, Farrow B, Awad S, Berger D and Albo D: Rectal cancer patients benefit from implementation of a dedicated colorectal cancer center in a Veterans Affairs medical center. Am J Surg 198: 100-104, 2009.

14. Pillay B, Wootten AC, Crowe H, Corcoran N, Tran B, Bowden P, Crowe $\mathrm{J}$ and Costello AJ: The impact of multidisciplinary team meetings on patient assessment, management and outcomes in oncology settings: A systematic review of the literature. Cancer Treat Rev 42: 56-72, 2016.

15. Ahmed HU, El-Shater Bosaily A, Brown LC, Gabe R, Kaplan R, Parmar MK, Collaco-Moraes Y, Ward K, Hindley RG, Freeman A, et al; PROMIS study group: Diagnostic accuracy of multi-parametric MRI and TRUS biopsy in prostate cancer (PROMIS): A paired validating confirmatory study. Lancet 389: 815-822, 2017.

16. Munro AJ: Multidisciplinary team meetings in cancer care: An idea whose time has gone? Clin Oncol (R Coll Radiol) 27: 728-731, 2015. 